\title{
QUALIDADE DE VIDA DOS TRABALHADORES DO CONTEXTO HOSPITALAR NA PANDEMIA DO CORONAVÍRUS
}

\section{André Vernier Stochero'; Karol Trevisan Sartori²; Jucimara Montagner Michelon $^{3}$; Tiago Durand Mussoi ${ }^{4}$; Cláudia Zamberlan ${ }^{5}$}

\section{RESUMO}

Investigar a qualidade de vida dos trabalhadores do contexto hospitalar na pandemia do Coronavírus. Pesquisa descritiva, exploratória de abordagem quantitativa. Os dados foram coletados pelo instrumento validado WHOQOL-BREF no primeiro semestre de $2021 \mathrm{com}$ os trabalhadores do contexto hospitalar que participam de um projeto de extensão intitulado "Cuide-se para Cuidar". Dos participantes, 18,75\% eram do sexo masculino e $81,25 \%$ do sexo feminino. Referente a aplicabilidade do instrumento na análise das duas primeiras facetas $62,5 \%$ avaliaram como boa a sua qualidade de vida e $53,12 \%$ estavam satisfeitos com sua saúde. As facetas três a 26, são referentes aos domínios físicos, psicológicos, relações sociais e ambiental. No domínio físico, $68,75 \%$ dos participantes estavam satisfeitos com sua qualidade de vida. No domínio psicológico $50 \%$ demonstram satisfação. Nas relações sociais, $62,5 \%$ também manifestaram satisfação e, no domínio ambiental, o nível de satisfação foi de $56,25 \%$. A qualidade de vida dos trabalhadores no contexto hospitalar durante a pandemia foi referenciada como satisfatória.

Palavras-chave: Covid 19; Hospital; Promoção da Saúde; Saúde laboral.

Eixo Temático: Atenção Integral e Promoção à Saúde (AIPS).

\section{INTRODUÇÃO}

No contexto atual, a mudança do perfil de saúde da população mundial, corrobora com mudanças, sobretudo, na utilização dos serviços de saúde, aumento de gastos com a mesma e, necessidade de evidências científicas que, articuladas às

\footnotetext{
1 Autor/Apresentador - Bolsista de Iniciação Científica PROBIC/UFN. Acadêmico do curso de Enfermagem da Universidade Franciscana (UFN). E-mail: andrevstochero@gmail.com

${ }^{2}$ Autora/Bolsista de Iniciação Científica PIBIC/CNPq - Acadêmica do Curso de Enfermagem da Universidade Franciscana (UFN). E-mail: karol.sartori@ufn.edu.br

${ }^{3}$ Autora/Mestranda em Saúde Materno Infantil. Universidade Franciscana (UFN). E-mail: enfer.jucimara@gmail.com

${ }^{4}$ Autor/Coorientador - Docente do Curso de Nutrição. Universidade Franciscana. E-mail: tdmussoi@gmail.com

${ }^{5}$ Autora/Orientadora - Docente do curso de Enfermagem. Universidade Franciscana (UFN). E-mail: claudiaz@ufn.edu.br
} 
tecnologias de cuidado fomentem ações em saúde eficazes à qualidade de vida das pessoas, grupos e população em geral.

Estes aspectos integrados e interconectados proporcionam desafios para o contexto de saúde nacional e internacional com necessidade de ações por meio de políticas de saúde que subsidiem as rápidas mudanças do perfil epidemiológico vigente. Referente as infecções transmissíveis, as doenças infecciosas e parasitárias têm grande importância para a saúde pública por estarem diretamente associadas à pobreza e a condições de vida inadequadas.

Esse cenário diversificado, torna complexo o trabalho em saúde, em especial, nos serviços de vigilância no que se refere à coleta, consolidação, avaliação e disseminação de informações para subsidiar a tomada de decisão, exigindo muitas inovações, sobretudo, na forma de articular as diferentes realidades. Nesse contexto, a identificação de áreas críticas para doenças infecciosas no Brasil, e, o conhecimento sobre a sua relação com indicadores socioeconômicos são fundamentais para alinhar as ações em âmbito local e nacional, fornecendo subsídios para o estabelecimento de medidas assertivas de controle, planejamento e intervenção, bem como articulações intersetoriais (SOUZA et al., 2020).

No final de 2019 surgiu um momento caótico no cenário internacional, a pandemia do covid-19, no qual, o provável foco inicial da contaminação em casos de pessoas infectadas com o coronavírus aconteceu na cidade de Wuhan na China onde trabalhadores e clientes de um mercado atacadista de frutos do mar se contaminaram pelo manuseio de animais vivos (FILHO et al., 2020).

O risco de contaminação pela doença é o principal problema de saúde que afeta os profissionais que se envolvem diretamente no cuidado aos pacientes com sintomas ou já diagnosticados com a covid-19. Esse risco tem causado afastamento do trabalho, doença, morte e também intenso sofrimento psíquico, o qual se manifesta por meio do transtorno de ansiedade generalizada, distúrbios do sono, medo de adoecer e de contaminar os colegas e familiares (TEIXEIRA et al., 2020).

A experiência dos profissionais de saúde no combate à nova pandemia de coronavírus integra as funções básicas de saúde pública, tendo como alvo pessoas ou grupos com maior risco de infeção, com foco nos profissionais de saúde. A 
EDUCAÇÃO, SAÚDE

ETECNOLOGIA

26 A 28 DE OUTUBRO DE 2021

atividade laboral e o trabalhador devem considerar e se preparar, não só para a proteção nos ambientes de trabalho, mas, além disso, compreender que suas atividades desempenham aspectos relevantes no combate à pandemia (FILHO et al., 2020).

No que se refere à saúde do trabalhador, ao mesmo tempo em que o País busca ampliação de recursos e infraestrutura de modo a obter melhores condições assistenciais no ambiente hospitalar, torna-se necessário, desenvolver ações voltadas a saúde dos trabalhadores que assumem o protagonismo no combate ao Coronavírus e após isto investigar como estas ações maximizam a qualidade de vida dos trabalhadores.

Para se evitar ou minimizar os riscos de infecção pelo covid-19, foram adotados protocolos se segurança no cenário nacional e internacional como: uso de máscaras, uso de álcool gel, lavagem das mãos e a manutenção do distanciamento social. Houve a necessidade da expansão de infraestrutura de leitos hospitalares e, também, a organização do processo de trabalho na atenção básica, salientando os critérios necessários para a proteção e a promoção da saúde física e mental dos profissionais e trabalhadores da saúde (TEIXEIRA et al., 2020).

Destaca-se ainda que a área da Saúde do Trabalhador é elencada como prioridade na Agenda de Prioridades de Pesquisa do Ministério da Saúde. Em consonância com esta prioridade e no intuito de atender uma demanda frente a pandemia de Covid-19, referente à Atenção à Saúde do Trabalhador, justifica-se essa pesquisa, pois, a partir das ações do Projeto de Extensão intitulado: "Cuide-se para cuidar" desenvolvido pelos Programas de Residência Multiprofissional da Universidade Franciscana (UFN) articulado à Medicina do Trabalho de um contexto hospitalar, foi possível verificar a qualidade de vida dos trabalhadores em tempo de pandemia referente as atividades desenvolvidas pelo projeto.

\section{OBJETIVO}

Investigar a qualidade de vida dos trabalhadores do contexto hospitalar na pandemia do Coronavírus. 


\section{METODOLOGIA}

Trata-se de um estudo descritivo, exploratório de abordagem quantitativa. Os dados foram coletados por meio de um instrumento validado denominado WHOQOL-BREF, com os trabalhadores de um hospital público de médio porte localizado na região central do Rio Grande do Sul, que participam de um projeto de extensão intitulado: Cuide-se para Cuidar.

O instrumento de coleta de dados denominado WHOQOL-BREF é composto por 26 questões, que abrangem perguntas sobre qualidade de vida a respeito da saúde e outras áreas da vida. Compreende quatro domínios da qualidade de vida, sendo que cada domínio tem por objetivo analisar, respectivamente: a capacidade física, o bem-estar psicológico, as relações sociais e o meio-ambiente onde a pessoa está inserida.

O primeiro participante que respondeu o questionário foi convidado pelos pesquisadores, a partir desse momento o primeiro participante indicou outro profissional participante do projeto e, assim sucessivamente, até atingir o número de 30 profissionais, que se configurou como o $\mathrm{n}$ amostral mínimo para a aplicação do instrumento.

Os dados foram coletados no período de maio a julho de 2021 e o convite para a participação realizado por e-mail e/ou contato telefônico, conforme disponibilidade dos participantes. Após esse momento e aceite, o questionário WHOQOL-BREF e, o termo de consentimento livre e esclarecido foi entregue pessoalmente pelo pesquisador ao profissional que aceitou participar da pesquisa. Os dados referentes às facetas do instrumento foram analisados por meio da estatística descritiva e, transcritos para uma planilha do Excel, a fim de realizar as análises das variáveis primárias. Para a realização desta pesquisa foram seguidos os aspectos éticos de pesquisa com seres humanos, conforme a Resolução 466/12 do Conselho Nacional de Saúde (BRASIL, 2012) e aprovada sob protocolo CAAE: 4.489.669.

\section{RESULTADOS E DISCUSSÕES}


O perfil dos participantes da pesquisa englobou diversos trabalhadores de diferentes setores participantes do projeto "Cuide-se para Cuidar", entre eles: enfermeiros, técnicos de enfermagem, auxiliar e assistente administrativo, costureira, atendente de lavanderia, terapeuta ocupacional, porteira, psicólogo, auxiliar de logística, gerente, assistente social e técnico de segurança do trabalho, tendo no total de 32 pessoas que responderam ao questionário. Destes 18,75\% (6) foram do gênero masculino e $81,25 \%$ (26) do gênero feminino.

Entende-se a qualidade de vida no trabalho como o bem-estar em sua conjuntura completa, ou seja, com o ambiente e com as demais pessoas que convive. Por isso, a gestão dos cenários hospitalares mantém um cuidado especial nas estratégias de organização ao perceber a importância do trabalhador dentro das instituições e como o bem-estar dele é expressivo para o aperfeiçoamento das tarefas no dia a dia, colaborando no crescimento da organização ou empresa e na saúde física e emocional do indivíduo que acaba sendo a mais afetada (MARTINS; ALVES; DIAS, 2020).

Referente a aplicabilidade do instrumento WHOQOL-BREF na análise das duas primeiras facetas $62,5 \%$ (20) avaliaram como boa a sua qualidade de vida e $53,12 \%$ (17) estavam satisfeitos com sua saúde. Das facetas de número três a 26 , a análise foi realizada por meio de domínios quais sejam: físicos, psicológicos, relações sociais e ambientais. No domínio físico, 68,75\% (22) dos participantes estavam satisfeitos com sua qualidade de vida, já no domínio psicológico 50\% (16) demonstram satisfação, no domínio de relações sociais, 62,5\% (20) também manifestaram satisfação. E por fim, o domínio ambiental, na qual o nível de satisfação foi configurado 56,25\% (18).

Estudo realizado com profissionais de programas de residência multiprofissional que atuam no contexto hospitalar e que utilizaram o instrumento WHOQOL-BREF verificou que para os dois primeiros domínios, os resultados são considerados satisfatórios e nos dois últimos apesar de alcançarem valores mais baixos, também foram considerados aceitáveis (ZANEI; OLIVEIRA e WHITAKER, 2019). 
Ainda, em pesquisa realizada com profissionais de um hospital público e que avaliou a qualidade de vida dos mesmos demonstrou que os mesmos enfatizaram que aproveitam muito suas vidas, e que, estavam satisfeitos consigo mesmos e com sua aparência física. Relataram, manter boa concentração diante das inúmeras dificuldades diárias. Entretanto, na questão que avalia a frequência de sentimentos negativos demonstrou que 9\% apresentavam esses sintomas frequentemente e 6,3\% muito frequentemente (QUEIROZ, FERREIRA, AZEVEDO, 2019).

Ainda, destaca-se conforme estudo de Santos; Paiva e Spiri (2018) que os fatores no ambiente hospitalar que influenciam na qualidade de vida dos profissionais de enfermagem são: exposição aos danos físicos e emocionais, recursos materiais, deficitários, sobrecarga e acidentes de trabalho, falta de valorização e reconhecimento profissional, descontentamento ambiental, dimensionamento de pessoal insuficiente.

\section{CONCLUSÃO}

Esta pesquisa demonstrou que a qualidade de vida dos trabalhadores no contexto hospitalar durante a pandemia, e que aceitaram participar da mesma foi referenciada como satisfatória. Desse modo, pode-se ressaltar a importância do cuidado com os profissionais atuantes no contexto hospitalar, visto que muitos, não tem tempo de desempenharem atividades diferentes em sua rotina de trabalho, e também por conta dos decretos impostos pela pandemia e pelo medo da contaminação.

Assim, por meio do projeto Cuide-se para Cuidar os mesmos obtiveram a oportunidade de frequentarem uma atividade diferente na rotina, porém, dentro do local de trabalho, com todo o cuidado necessário, visando manter uma boa qualidade de vida.

Este apoio aos trabalhadores possibilitou uma melhor qualidade de vida, com menos afastamento dos seus serviços e melhor aperfeiçoamento na prestação de seu trabalho para seu setor dentro do hospital. Por fim, destaca-se que o projeto de extensão "Cuide-se para Cuidar" que está sendo desenvolvido nesta instituição é de grande relevância e está contribuindo com esta métrica. 


\section{AGRADECIMENTOS}

Agradeço à minha orientadora, coorientador e demais autores por me ajudarem na construção deste material. A bolsa de estudos PROBIC - UFN, por proporcionar este momento e a Universidade Franciscana (UFN).

\section{REFERÊNCIAS}

BRASIL. Ministério da Saúde. Conselho Nacional de Saúde. Resolução 466/2012. Resolução para pesquisas envolvendo seres humanos. Brasília, DF: Ministério da Saúde, 2012.

FILHO, J. M. J.; ASSUNÇÃO, A. A.; ALGRANTI, E.; GARCIA, E. G.; SAITO, C. A.; MAENO, M. A saúde do trabalhador e o enfrentamento da COVID-19. Rev. bras. saúde ocup. v. 45, n. 14 p. 01-03, 2020. Disponível em: https://www.scielo.br/scielo.php?script=sci_arttext\&pid=S0303-76572020000100100 Acesso em: 05 jun. 2021.

MARTINS, K. F.; ALVES, M. S.; DIAS, A. K. Qualidade de vida no ambiente hospitalar dos profissionais de enfermagem. Revista Amazônia Science \& Health. V. 8, n. 1, p. 37-54, 2020. Disponível em: http://ojs.unirg.edu.br/index.php/2/article/view/3085/1577. Acesso em: 10 ago. 2021.

QUEIROZ, B. R. S.; FERREIRA, M. G.; AZEVEDO, O. A. Qualidade de vida do profissional de enfermagem que atua em uma instituição hospitalar da rede pública. LifeStyle Journal, São Paulo, v. 6, n. 1, p. 31-46, 2019.

SANTOS, R. R.; PAIVA, M. C. M. S.; SPIRI, W. C. Associação entre qualidade de vida e ambiente de trabalho de enfermeiros. Acta Paul Enferm. V. 31, n. 5, p. 472479, 2018. Disponível em: https://www.scielo.br/j/ape/a/XV73M3N6B34FMb3QXtsbMGc/?format=pdf\&lang=pt. Acesso em: 10 ago. 2021. 


\section{QUFN}

SOUZA, H. P.; OLIVEIRA W. T. G. H.; SANTOS, J. P. C.; TOLEDO, J. P.; FERREIRA, I. P. S.; ESASHIKA, S. N. G. S.; LIMA, T. F. P.; DELÁCIO, A. S. Doenças infecciosas e parasitárias no Brasil de 2010 a 2017: aspectos para vigilância em saúde. Rev. Panam Salud. Publica. V. 44. p. 01-07, 2020. Disponível em:

https://iris.paho.org/bitstream/handle/10665.2/51858/v44e102020.pdf?sequence=1\&i sAllowed=y. Acesso em: 05 jun. 2021.

TEIXEIRA, C. F. S.; SOARES, C. M.; SOUZA, E. A.; LISBOA, E. S.; PINTO, I. C. M.; ANDRADE, L. R.; ESPIRIDIÃO, M. A. A saúde dos profissionais de saúde no enfrentamento da pandemia de Covid-19. Ciênc. saúde coletiva. V. 25, n. 9, p. 3465-3469, 2020. Disponível em:

https://www.scielo.br/j/csc/a/6J6vP5KJZyy7Nn45m3Vfypx/?format=pdf\&lang=pt. Acesso em: 08 ago. 2021.

ZANEI, S. S. V.; OLIVEIRA, R. A. O.; WHITAKER, I. Y. Qualidade de vida dos profissionais de saúde dos programas de residências multidisciplinares. Rev. Enferm. UFSM - REUFSM. Santa Maria, RS, v. 9, e35, p. 1-20, 2019.Disponível em: https://periodicos.ufsm.br/reufsm/article/view/30013/html. Acesso em: 15 ago. 2021. 